Actas del Seminario Internacional Destinos Turísticos Inteligentes:

nuevos horizontes en la investigación y gestión del turismo

Universidad de Alicante, 26 y 27 de octubre de 2017

\title{
La relativa importancia de la velocidad en las conexiones WIFI
}

\author{
Juan Pedro Mellinas \\ Universidad Internacional de La Rioja, España \\ Facultad de Ciencias Jurídicas, Sociales y Humanidades \\ losmellinas@yahoo.es
}

\begin{abstract}
Los turistas quieren acceder a una conexión WiFi desde que llegan a sus destinos, para evitar sobrecargos en sus facturas telefónicas. Entidades públicas y privadas ofrecen este servicio en todo el mundo, pero los turistas suelen quejarse cuando es lento o poco fiable. La velocidad parece ser el factor más importante que determina el nivel de satisfacción de los viajeros con el WiFi, por lo que testamos esta hipótesis centrándonos en los hoteles. Los datos sobre velocidad de conexión son proporcionados por Hotelwifitest.com y los datos sobre satisfacción se extraen de Booking. com. Los resultados muestran que existe una relación muy débil entre ambas variables, por lo que se hace necesaria una investigación adicional para encontrar otros factores que influyan en la satisfacción con el servicio de WiFi.
\end{abstract}

Palabras clave: Wifi, hotel, destino, velocidad, satisfacción.

\begin{abstract}
Tourists just want to access WiFi when they arrive to a destination, in order to avoid charges in their telephone bills. Public and private organizations provide this service all around the world, but tourist complaint when it is slow or not reliable. Speed seems to be the most important factor determining the level of satisfaction of travellers with WiFi, so this hypothesis is tested, focusing in hotels. Speed data are provided by Hotelwifitest.com and satisfaction data by Booking.com. Results show that there is a very weak relation between both factors, so additional research should be done to identify other relevant factors affecting satisfaction about WiFi.
\end{abstract}

Keywords: Wifi, hotel, destination, speed, satisfaction. 
Actas del Seminario Internacional Destinos Turísticos Inteligentes:

nuevos horizontes en la investigación y gestión del turismo

Universidad de Alicante, 26 y 27 de octubre de 2017

\section{Introducción}

Vivimos en un mundo interconectado, en el que no se puede prescindir del acceso a Internet, razón por la que consideramos el WiFi como algo imprescindible en nuestros viajes, dado que los límites de las tarifas de datos contratadas pueden agotarse rápidamente si se prescinde del acceso WiFi durante unos días. Esta dependencia se acentúa cuando el viaje se produce fuera del país, lo que implica importantes cargos por «roaming» en la conexión personal a Internet. Es algo parcialmente subsanado con la reciente normativa europea que evita estos sobrecostes (European Commission, 2016) para viajes dentro de la UE, pero que seguirá siendo un problema para el resto de visitantes, especialmente con los procedentes de Reino Unido, el principal emisor de turistas hacia España.

Los viajeros esperan tener una conexión Wifi adecuada a lo largo de su estancia, comenzando en los aeropuertos y continuando en centros de visitantes, restaurantes, museos y otras atracciones. Pero, sin duda, es en el alojamiento, donde el usuario espera contar con una conexión Wifi adecuada, ya que es donde más tiempo pasará.

Si bien hace unos años la conexión WiFi gratuita era algo que solo ofrecían algunos hoteles, actualmente la gran mayoría lo hacen, aunque en algunos casos con una velocidad de conexión limitada, dejando como una opción "de pago» la conexión de alta velocidad. Si bien una conexión básica (inferior a $1 \mathrm{Mbps}$ ) puede ser suficiente para acceder a redes sociales, revisar el correo o leer noticias; en el momento en que pretendemos visualizar vídeos, utilizar Skype, trabajar con programas en la nube o descargar archivos, se hace necesaria una conexión con velocidad superior.

Dado que la práctica totalidad de los hoteles ofrecen una conexión WiFi gratuita, las diferencias en este aspecto vienen determinadas por la mayor o menor calidad del servicio. Nuestra hipótesis inicial plantea que el principal factor que influye en esa percepción de calidad por parte de los usuarios es la velocidad de la conexión. Partimos de la hipótesis de que a mayor velocidad de conexión obtendremos mayor satisfacción del cliente, llegando un punto en el que aumentos de velocidad, no produzcan variaciones significativas en la satisfacción.

Para medir la satisfacción con el servicio de WiFi gratuito, tomamos la información de las opiniones de los viajeros en Booking.com (Booking), ya que tiene un apartado específico en el que sus clientes valoran este servicio. 
Actas del Seminario Internacional Destinos Turísticos Inteligentes:

nuevos horizontes en la investigación y gestión del turismo

Universidad de Alicante, 26 y 27 de octubre de 2017

Mientras que para obtener datos de la velocidad de conexión, obtenemos la información de la web hotelwifitest.com que recopila test de velocidad de conexión en hoteles, realizados por usuarios.

Consideramos que las conclusiones obtenidas para el caso del WiFi de los hoteles, pueden ser trasladadas al resto de establecimientos que proveen WiFi a los viajeros. De esta forma podremos determinar si deben centrarse en incrementar la velocidad de sus conexiones o si, por el contrario, deberían mejorar otros aspectos de las conexiones ofrecidas.

\section{Revisión bibliográfica}

Hemos pasado en pocos años de considerar el Wifi gratuito en los hoteles como un elemento diferenciador (Bulchand-Gonzalez, 2011; Lee \& Tussyadiah, 2010), a ser visto como un servicio básico, que ofrecen la gran mayoría de los hoteles en el mundo (The Telegraph, 2014; Tripbarometer, 2015; AHLA, 2016).

Los estudios de los últimos 3 años sitúan la conexión Wi-Fi gratuita de los hoteles como un factor de suma importancia en la satisfacción que los clientes muestran sobre su estancia. Se ha situado como la "amenitie» más importante para los huéspedes a la hora de elegir un hotel (Tripbarometer, 2015) especialmente para los viajeros de negocios (Brochado et al., 2016). De hecho un estudio sobre viajeros de Estados Unidos indicó que el WiFi es el segundo factor más importante al elegir un hotel, por encima del desayuno o el parking, únicamente superado por el precio (Red Roof, 2016). En un estudio similar con viajeros británicos, el $60 \%$ lo volvió a señalar como el factor más importante, por encima de un sueño de calidad (58\%) y del trato recibido por el personal del hotel (40\%) (Amba Hotels, 2014).

La conexión WiFi gratuita es el factor más importante para los viajeros de ocio (30\%), mientras que el $50 \%$ de los viajeros de negocios lo consideran como algo imprescindible en sus desplazamientos de trabajo (ehotelier, 2015). Casi la mitad de los viajeros de negocios que no están satisfechos con su estancia (46\%) identifican la mejora de la conexión WiFi como el factor que más habría ayudado a mejorar su experiencia (GBTA, 2016), además manifiestan que no tener una conexión Wifi adecuada es el aspecto más frustrante de sus viajes de trabajo (25\%). Un artículo del diario británico Daily Mail (Pemberton, 2016) trataba este tema, usando el título «Forget diving into the infinity pool or rushing to the beach: Hotel guests just want to access Wi-Fi when they arrive (with 65\% logging on within SEVEN minutes)» 
Actas del Seminario Internacional Destinos Turísticos Inteligentes:

nuevos horizontes en la investigación y gestión del turismo

Universidad de Alicante, 26 y 27 de octubre de 2017

(Olvídense de nadar en piscinas infinitas o de correr a la playa: los huéspedes quieren acceso al WiFi en cuanto llegan, con el $65 \%$ registrándose en los primeros siete minutos).

Las opiniones de los viajeros sobre su experiencia con el WiFi en los hoteles que visitan, en webs como TripAdvisor, Booking o Expedia, nos proporcionan una información importante sobre sus niveles de satisfacción con este servicio. Los sistemas de recomendación entre usuarios han existido desde hace muchos años en el sector turístico, incluso antes de la aparición de internet (Butler, 1980; Cohen, 1972). Es lo que se conoce en castellano como «boca a boca» o «boca a oreja», cuyo equivalente en inglés sería «word of mouth» (WOM), que es la denominación por la que es conocido este fenómeno en el ámbito académico.

Con la irrupción de Internet a finales del siglo XX y especialmente con la posibilidad de que los usuarios aporten contenidos que proporciona la llamada Web 2.0 (O'Reilly, 2005) a principios del siglo XXI, aparecen los comentarios y opiniones sobre productos y servicios. Se acuña así el término "electronic Word of mouth» (eWOM) para referirse a las comunicaciones entre usuarios, a través de Internet, que versan sobre el uso de productos y servicios, o sus distribuidores (Litvin et al., 2016). El eWOM tiene una capacidad de influencia muy superior al tradicional WOM, principalmente por su velocidad de propagación, facilidad de acceso y la libertad que proporciona el anonimato (Sun et al., 2006).

El eWOM tiene un impacto significativo en las actitudes de los consumidores y en sus intenciones de compra (Park et al., 2007; Doh and Hwang, 2009; Ladhari and Michaud, 2015), lo que afecta directamente a los resultados de ventas de las empresas (Chevalier and Mayzlin, 2006; Liu, 2006; Dellarocas et al., 2007; Cheung et al., 2009). Cuando nos referimos al sector turístico, diversos estudios han demostrado que estas opiniones influyen en la planificación de los viajes y en las decisiones de contratación de servicios (Gretzel and Yoo, 2008; Vermeulen and Seegers, 2009). Los consumidores tienden a confiar más en las opiniones de desconocidos en Internet que en las campañas de publicidad y marketing de las empresas turísticas (Browning, So, \& Sparks, 2013; Ricci \& Wietsma, 2006).

Aunque, como se ha señalado, los viajeros consideran imprescindible una conexión Wifi de calidad en el hotel, también desean tener acceso a este tipo de conexiones en otras ubicaciones. Las esperas en los aeropuertos resultan especialmente proclives a la demanda de este servicio y su ausencia 
Actas del Seminario Internacional Destinos Turísticos Inteligentes:

nuevos horizontes en la investigación y gestión del turismo

Universidad de Alicante, 26 y 27 de octubre de 2017

o falta de fiabilidad causa ciertos niveles de frustración (Batra, 2014). Así mismo los restaurantes se han convertido en lugares en los que se espera una conexión WiFi gratuita, convirtiéndose incluso en un importante elemento para la captación y fidelización de clientes (Cobanoglu et al., 2014).

Las entidades turísticas a nivel municipal también son potenciales proveedores de conexiones WiFi gratuitas para satisfacer esta demanda por parte de los turistas. A principios de este siglo, junto a la popularización de la tecnología WiFi, muchas ciudades en todo el mundo decidieron implantar redes públicas gratuitas para el acceso a Internet, dirigidas tanto a empleados municipales, como a los propios ciudadanos y turistas (Middelton, 2007). Pero la euforia inicial en la implantación de estos servicios se ha visto limitada por diferentes condicionantes, entre los que destaca el hecho de que estos servicios entraban a competir con los servicios de pago de las compañías de telefonía (Picco-Schwendener \& Cantoni, 2015). En todo caso, siguen existiendo este tipo de conexiones en muchas ciudades del mundo, principalmente orientadas a ofrecer este servicio a los turistas, aunque generalmente limitadas a espacios públicos concretos como museos u oficinas de turismo.

\section{Metodología y objetivos}

Los hoteles no facilitan la velocidad exacta de su conexión WiFi. De hecho esta velocidad puede variar, dependiendo del número de usuarios conectados en cada momento o la ubicación del usuario dentro del establecimiento. Para tener una estimación de este valor es posible recurrir a webs que permiten a los usuarios realizar test de velocidad en los hoteles en los que se alojan. Estos datos son recopilados y mostrados públicamente, lo que proporciona una valiosa información a los viajeros preocupados por la velocidad que puede proporcionar cada hotel.

Entre estas webs identificamos principalmente Hotelwfitest.com (Figura 1) y Wifirank.com, que aparentemente proporcionan datos similares. Pero al analizar la base de datos de ambas webs, observamos como la cantidad de hoteles testados en Hotelwifitest.com es muy superior a la de Wifirank.com. Por ejemplo encontramos 298 hoteles testados en París para una, mientras que la otra tiene únicamente 9 , algo similar a lo que sucede en el caso de Londres con 450 y 41 hoteles testados respectivamente. Estas diferencias nos hacen inclinarnos por Hotelwifitest.com que, aunque ofrece a los hoteles herramientas de pago para mejorar la calidad de su red Wifi, manifiesta 
Actas del Seminario Internacional Destinos Turísticos Inteligentes:

nuevos horizontes en la investigación y gestión del turismo

Universidad de Alicante, 26 y 27 de octubre de 2017

que la totalidad de los datos proporcionados provienen de test reales realizados en el hotel por clientes reales.

Figura 1. Datos de conexión a un hotel en Hotelwifitest.com

W12 Rooms
54 Uxbridge Road, London W12 8LP, United Kingdom

Fuente: www.hotelwifitest.com

Por otra parte, los usuarios comparten sus opiniones sobre el servicio de WiFi de los hoteles a través de webs como TripAdvisor, Expedia, Agoda o Booking. En este último caso, la información proporcionada no se limita a los comentarios de los usuarios, sino que se cuantifica el nivel de satisfacción, proporcionando una puntuación sobre el WiFi en los hoteles que ofrecen este servicio, al igual que se hace sobre otros aspectos como la limpieza, el servicio o la ubicación (Figura 2). Booking recoge una cantidad de opiniones sobre hoteles superior al de otras web, acumulando el $39 \%$ del total de las opiniones online sobre hoteles (Revinate, 2017).

Figura 2. Ejemplo de puntuaciones de un hotel en Booking.com

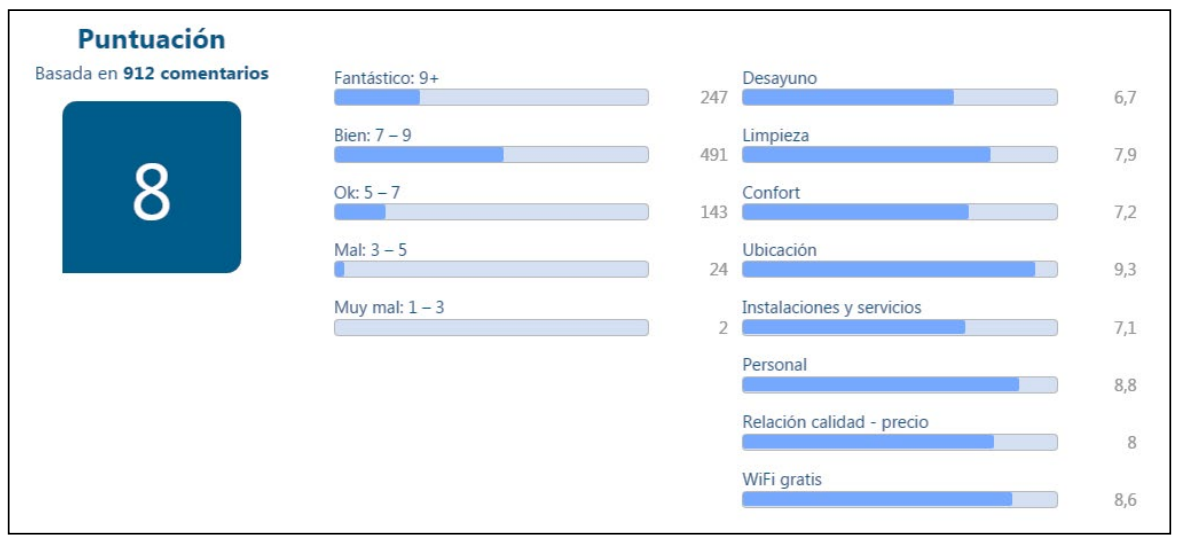

Fuente: www.booking.com 
Actas del Seminario Internacional Destinos Turísticos Inteligentes:

nuevos horizontes en la investigación y gestión del turismo

Universidad de Alicante, 26 y 27 de octubre de 2017

Para testar la hipótesis planteada tomamos datos de una muestra de hoteles testados adecuadamente en HotelWifiTest, que además comercializan sus servicios a través de Booking. Para seleccionar los hoteles en HotelWifiTest tenemos en cuenta aquellos cuya ficha indique un nivel de confianza mínimo del $40 \%$. Este nivel depende de varios factores, incluido el número de test realizados y si estos son más o menos recientes. La muestra inicial está constituida por la totalidad de los hoteles que cumplen este requisito, pertenecientes a las 6 ciudades con más hoteles testados en esta web (Londres, Nueva York, Paris, Bangkok, Berlín y Barcelona). De esta muestra inicial se extraen únicamente los que comercializan sus servicios en Booking, ofrecen WiFi gratuito y tienen al menos 200 opiniones registradas. Más del $90 \%$ de los hoteles de la muestra inicial se incluyen en la muestra final, al disponer de datos adecuados en Booking, con lo que obtenemos una muestra final de 200 alojamientos.

\section{Resultados}

Al realizar una regresión lineal, los resultados obtenidos muestran que, efectivamente, a mayor velocidad de conexión el nivel de satisfacción de los usuarios con el WiFi es superior. Sin embargo el nivel de relación entre ambas variables es muy bajo, así como la calidad del ajuste (Tabla 1). Al dividir la muestra en cuartiles (en función de la velocidad) observamos como para el segundo y tercer cuartil, existe incluso una relación inversa entre ambas variables. Además, apenas hay diferencias entre las puntuaciones medias de los diferentes cuartiles, incluso observamos anomalías como una media inferior en el tercer cuartil respecto al segundo y una mediana idéntica en el primer y segundo cuartil. 
Actas del Seminario Internacional Destinos Turísticos Inteligentes:

nuevos horizontes en la investigación y gestión del turismo

Universidad de Alicante, 26 y 27 de octubre de 2017

Tabla 1. Datos por cuartil y totales

\begin{tabular}{|c|c|c|c|c|c|c|}
\hline CUARTIL & $\begin{array}{c}\text { Media } \\
\text { Velocidad }\end{array}$ & $\begin{array}{c}\text { Mediana } \\
\text { Velocidad }\end{array}$ & $\begin{array}{c}\text { Media } \\
\text { puntuación }\end{array}$ & $\begin{array}{c}\text { Mediana } \\
\text { puntuación }\end{array}$ & $\mathbf{R}^{\mathbf{2}}$ & y \\
\hline 10 Cuartil & 1,54 & 1,50 & 8,15 & 8,30 & 0,005 & $0,0573 x+8,0619$ \\
\hline 20 Cuartil & 4,66 & 4,65 & 8,30 & 8,30 & 0,0014 & $-0,0212 x+8,3946$ \\
\hline 3o Cuartil & 11,35 & 10,60 & 8,26 & 8,35 & 0,0088 & $-0,0161 x+8,4423$ \\
\hline 40 Cuartil & 42,01 & 27,40 & 8,59 & 8,60 & 0,1172 & $0,005 x+8,3818$ \\
\hline TOTAL & $\mathbf{1 4 , 8 9}$ & $\mathbf{6 , 6 5}$ & $\mathbf{8 , 3 2}$ & $\mathbf{8 , 4 0}$ & $\mathbf{0 , 0 7 9}$ & $\mathbf{0 , 0 0 6 9 x}+\mathbf{8 , 2 2 1 2}$ \\
\hline
\end{tabular}

Fuente: Elaboración propia a partir de datos de www.hotelwifitest.com y www.booking.com

Se representan los datos obtenidos sobre una gráfica, en la que cada punto representa a uno de los 200 hoteles de la muestra (Figura 3). Observamos como los hoteles con altas velocidades (mayores de $30 \mathrm{Mbps}$ ) no presentan puntuaciones significativamente por debajo de la media, pero también detectamos que muchos hoteles con velocidades por debajo de la media, logran obtener puntuaciones de alrededor de 9 puntos (muy por encima de la media).

Figura 3. Regresión lineal de la muestra de hoteles

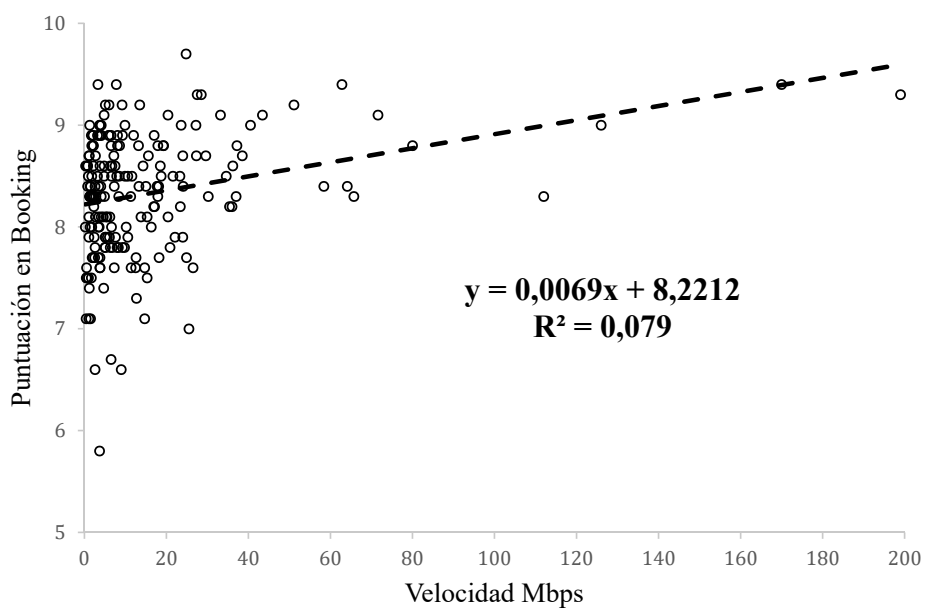

Fuente: Elaboración propia a partir de datos de www.hotelwifitest.com y www.booking.com 
Actas del Seminario Internacional Destinos Turísticos Inteligentes:

nuevos horizontes en la investigación y gestión del turismo

Universidad de Alicante, 26 y 27 de octubre de 2017

Al observar la representación gráfica observamos como la mayor parte de los datos se concentran en el tramo inferior de velocidad (0-40 Mbps), con únicamente 10 valores en el tramo (40-200 Mbps). Sospechamos que esos 10 valores superiores podrían considerarse como "outliers" y estarían distorsionando los resultados obtenidos, impidiendo observar la buscada relación entre las dos variables estudiadas. Eliminamos esos 10 valores quedando una muestra de 190 hoteles, sobre la que realizamos el mismo análisis (Figura 4). Esto nos permite observar de una forma más clara la distribución de las observaciones, pero sigue mostrando una relación muy débil entra ambas variables.

Figura 4. Regresión lineal eliminando los 10 hoteles con mayor velocidad

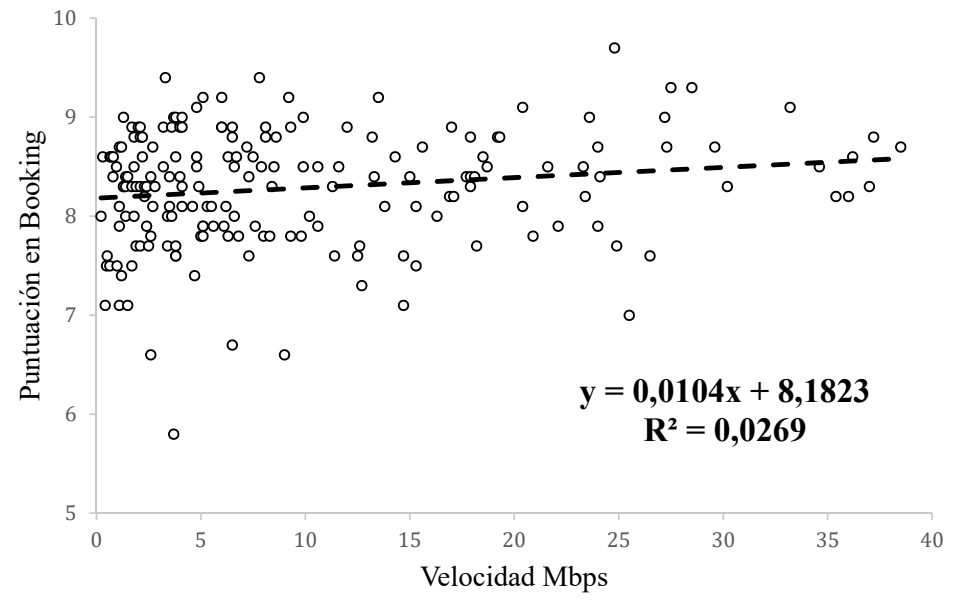

Fuente: Elaboración propia a partir de datos de www.hotelwifitest.com y www.booking.com

Al desagregar los datos por ciudad, observamos diferencias en las velocidades medias, incluso en las puntuaciones obtenidas en cada ubicación (Tabla 2). Pero seguimos sin observar una clara relación entre velocidad de la conexión WiFi y puntuaciones asignadas por los usuarios. Incluso en dos de las ciudades (Berlín y Barcelona), los datos indican una ligera relación inversa entre ambas variables. 
Actas del Seminario Internacional Destinos Turísticos Inteligentes:

nuevos horizontes en la investigación y gestión del turismo

Universidad de Alicante, 26 y 27 de octubre de 2017

Tabla 2. Datos por ciudad

\begin{tabular}{|c|c|c|c|c|c|c|c|}
\hline CIUDAD & $\begin{array}{c}\text { Media } \\
\text { Velocidad }\end{array}$ & $\begin{array}{c}\text { Mediana } \\
\text { Velocidad }\end{array}$ & $\begin{array}{c}\text { Media } \\
\text { puntuación }\end{array}$ & $\begin{array}{c}\text { Mediana } \\
\text { puntuación }\end{array}$ & $\begin{array}{c}\text { N.o } \\
\text { Hoteles }\end{array}$ & $\mathbf{R}^{\mathbf{2}}$ & $\mathbf{y}$ \\
\hline Bangkok & 7,50 & 5,95 & 7,97 & 8,00 & 22 & 0,0503 & $0,0292 x+7,7492$ \\
\hline Berlín & 9,16 & 6,15 & 8,17 & 8,20 & 52 & 0,0151 & $-0,0087 x+8,2545$ \\
\hline Londres & 26,04 & 15,00 & 8,52 & 8,50 & 49 & 0,2691 & $0,0066 x+8,3495$ \\
\hline N. York & 11,94 & 4,8 & 8,53 & 8,60 & 43 & 0,05 & $0,0070 x+8,4513$ \\
\hline París & 12,21 & 5,10 & 8,15 & 8,20 & 21 & 0,1573 & $0,0160 x+7,9523$ \\
\hline Barcelona & 22,37 & 10,2 & 8,37 & 8,30 & 13 & 0,002 & $-0,0006 x+8,3828$ \\
\hline
\end{tabular}

Fuente: Elaboración propia a partir de datos de www.hotelwifitest.com y www.booking.com

Rechazamos por lo tanto la hipótesis inicial, ya que los datos indican que la relación entre velocidad de conexión y satisfacción con el servicio WiFi, es mínima. Incluso en el caso de ciertos grupos de hoteles, parece no existir tal relación o ser inversa.

\section{Conclusiones}

Los datos obtenidos indican que el factor "velocidad", si bien es evidente que debe tener su importancia, no es el único condicionante que debería ser estudiado para determinar la calidad de una conexión WiFi pública. No solo se trata de que la velocidad sea adecuada, los usuarios deben ser capaces de disfrutar de una experiencia satisfactoria con su conexión WiFi. De no ser así, este servicio será valorado con puntuaciones bajas, lo que hará que baje sustancialmente la puntuación del hotel en este aspecto.

Contrariamente a lo esperado al formular la hipótesis inicial, parece muy complicado identificar una velocidad WiFi a partir de la cual los usuarios se muestren «muy satisfechos» de forma generalizada. La percepción de la experiencia se ve afectada por una serie de factores técnicos que afectan a la puntuación final que asigna el usuario. Uno de ellos sería la estabilidad de la conexión, evitando eventuales cortes que hacen inviable o incómodo utilizar ciertos servicios. Otros podrían ser la sencillez del proceso de autentificación y acceso, la cobertura en todas las dependencias del hotel o el tiempo de respuesta de la conexión (ping). En todo caso, sería necesario un 
Actas del Seminario Internacional Destinos Turísticos Inteligentes:

nuevos horizontes en la investigación y gestión del turismo

Universidad de Alicante, 26 y 27 de octubre de 2017

estudio más profundo que proporcionara un listado completo de factores y su importancia relativa.

Queda así claro que tratar de medir la calidad de una conexión WiFi, exclusivamente por la velocidad que puede llegar a proporcionar en una situación determinada, es un claro error. Estas conclusiones extraídas de datos sobre hoteles son aplicables a cualquier institución que pretenda ofrecer un servicio WiFi gratuito. Los museos, aeropuertos u oficinas de turismo que proporcionan WiFi a los visitantes, deberían realizar un análisis pormenorizado de la calidad de sus conexiones. De nada sirve un WiFi con una velocidad adecuada, si hay un número importante de turistas que no son capaces de conectarse, lo hacen de manera intermitente o que experimentan dificultades en el disfrute del servicio.

Como limitación principal de este estudio, podría señalarse la fiabilidad de los datos proporcionados por HotelWifiTest. La propia web reconoce que las velocidades proporcionadas pueden no ser del todo precisas por el número limitado de test realizados. También podría producir un sesgo el hecho de que los clientes que no lograron conectarse al WiFi gratuito del hotel no pueden realizar el test en esta web, pero probablemente se sentirán frustrados por esa mala experiencia y asignarán una puntuación baja al ítem "WiFi gratis» cuando rellenen la encuesta de Booking.

En este mismo sentido, las puntuaciones asignadas en Booking pueden ser poco útiles para ciertos usuarios, que podrían esperar que una valoración alta correspondiera a una velocidad alta. Vemos como hay muchos casos de hoteles con velocidades relativamente bajas que sin embargo obtienen puntuaciones relativamente altas en Booking.

A partir de estos datos, las nuevas investigaciones en este aspecto deberían ir encaminadas a establecer sistemas de auditoría de calidad de las conexiones $\mathrm{WiFi}$, que no se limiten a realizar un test con uno o dos dispositivos en una ubicación aleatoria de la zona con «supuesta» cobertura WiFi. Además deberían realizarse cuestionarios que lograran identificar los aspectos fundamentales que condicionan la percepción de calidad de una conexión WiFi, una vez demostrado que no resulta operativo considerar únicamente la velocidad. 
Actas del Seminario Internacional Destinos Turísticos Inteligentes:

nuevos horizontes en la investigación y gestión del turismo

Universidad de Alicante, 26 y 27 de octubre de 2017

\section{Referencias bibliográficas}

AHLA (2016). New survey reveals latest hotel trends across the country. Recuperado de: https://www.ahla.com/press-release/new-survey-reveals-latest-hoteltrends-across-country (21/02/2017)

Amba Hotels (2014). Research shows fast, free WI-FI is top of the wishlist for UK travelers. Recuperado de: http://www.amba-hotel.com/corporate/about_us/ press_office/research_shows_fast_free_wi-fi_is_top_of_the_wishlist_for_uk_ travellers.html (21/02/2017)

Batra, A. (2014). The role of airport servicescape: The transient community perspective. Tourism Dimensions, 1(1), 27-37.

Brochado, A., Rita, P., \& Margarido, A. (2016). High tech meets high touch in upscale hotels. Journal of Hospitality and Tourism Technology, 7(4), 347-365.

Browning, V., So, K. K. F., \& Sparks, B. (2013). The influence of online reviews on consumers' attributions of service quality and control for service standards in hotels. Journal of Travel \& Tourism Marketing, 30(1-2), 23-40.

Bulchand-Gidumal, J., Melián-González, S., \& González López-Valcárcel, B. (2011). Improving hotel ratings by offering free Wi-Fi. Journal of Hospitality and Tourism Technology, 2(3), 235-245.

Butler, R. W. (1980). The concept of a tourist area cycle of evolution: Implications for management of resources. The Canadian Geographer/Le Géographe Canadien, 24(1), 5-12.

Cheung, M.Y., Luo, C., Sia, C.L. and Chen, H. (2009). Credibility of electronic word-ofmouth: informational and normative determinants of on-line consumer recommendations. International Journal of Electronic Commerce, 13(4), 9-38.

Chevalier, J. A., \& Mayzlin, D. (2006). The effect of word of mouth on sales: Online book reviews. Journal of marketing research, 43(3), 345-354.

Cobanoglu, C., Bilgihan, A., Nusair, K. K., \& Berezina, K. (2012). The impact of Wi-Fi service in restaurants on customers' likelihood of return to a restaurant. Journal of Foodservice Business Research, 15(3), 285-299.

Cohen, E. (1972). Toward a sociology of international tourism. Social Research, 164182.

Dellarocas, C., Zhang, X. M., \& Awad, N. F. (2007). Exploring the value of online product reviews in forecasting sales: The case of motion pictures. Journal of Interactive marketing, 21(4), 23-45.

Doh, S. J., \& Hwang, J. S. (2009). How consumers evaluate eWOM (electronic wordof-mouth) messages. CyberPsychology \& Behavior, 12(2), 193-197.

Ehotelier (2015). What amenities guest want: free Wi-Fi still on top of survey. Recuperado de: https://ehotelier.com/global/2015/05/07/what-amenities-guestwant-free-wi-fi-still-on-top-of-survey/ (21/02/2017) 
Actas del Seminario Internacional Destinos Turísticos Inteligentes:

nuevos horizontes en la investigación y gestión del turismo

Universidad de Alicante, 26 y 27 de octubre de 2017

European Commission (2016). End of roaming charges for travellers in the EU in 2017. Recuperado de: http://europa.eu/rapid/press-release_MEMO-16-4396_ en.htm (21/02/2017)

GBTA (2016). GBTA Business Traveler Sentiment Index Global Report - June 2016. In partnership with American Express. Recuperado de: https://business.americanexpress.com/us/business-trends-and-insights/business-traveler/gbta-business-traveler-sentiment-index-june-2016 (21/02/2017)

Gretzel, U., \& Yoo, K. H. (2008). Use and impact of online travel reviews. Information and communication technologies in tourism 2008, 35-46.

Ladhari, R., \& Michaud, M. (2015). eWOM effects on hotel booking intentions, attitudes, trust, and website perceptions. International Journal of Hospitality Management, 46, 36-45.

Lee, G., \& Tussyadiah, I. P. (2010). The Influence of Wi-Fi Service on Hotel Customer Satisfaction. In Proceedings of the 9th Asia Pacific Forum for Graduate Students' Research in Tourism. Kyushu: Beppu.

Litvin, S. W., Goldsmith, R. E., \& Pan, B. (2008). Electronic word-of-mouth in hospitality and tourism management. Tourism management, 29(3), 458-468.

Liu, W., Guillet, B. D., Xiao, Q., \& Law, R. (2014). Globalization or localization of consumer preferences: The case of hotel room booking. Tourism Management, 41, 148-157.

Middleton, C. A. (2007). A framework for investigating the value of public wireless networks. Presented at the Telecommunications Policy Research Conference (TPRC). Arlington, VA.

O'Reilly, T. (2005). What is Web 2.0. Design Patterns and Business Models for the

Next Generation of Software. Recuperado de: http://www.oreilly.com/pub/a/web2/ archive/what-is-web-20.html (21/02/2017)

Park, D. H., Lee, J., \& Han, I. (2007). The effect of on-line consumer reviews on consumer purchasing intention: The moderating role of involvement. International journal of electronic commerce, 11(4), 125-148.

Pemberton, B. (2016). Forget diving into the infinity pool or rushing to the beach: Hotel guests just want to access Wi-Fi when they arrive (with $65 \%$ logging on within SEVEN minutes). Daily Mail (29 March 2016). Recuperado de: http:// www.dailymail.co.uk/travel/travel_news/article-3513582/Forget-diving-infinity-pool-rushing-beach-Hotel-guests-just-want-access-Wi-Fi-arrive-65-loggingSEVEN-minutes.html\#ixzz4Wx68Gabs (21/02/2017)

Picco-Schwendener, A., \& Cantoni, L. (2015). Tourists and Municipal Wi-Fi Networks (MWN): The Case of Lugano (Switzerland). In Information and Communication Technologies in Tourism 2015 (pp. 565-578). Springer International Publishing. 
Actas del Seminario Internacional Destinos Turísticos Inteligentes:

nuevos horizontes en la investigación y gestión del turismo

Universidad de Alicante, 26 y 27 de octubre de 2017

Red Roof (2016). Red Roof survey says: WIFI more important than breakfast and parking. Recuperado de: https://www.redroof.com/about_us/announcements/ index.cfm?task=detail\&id=PR_16080108353012_ef5k7 (21/02/2017)

Ricci, F., \& Wietsma, R. T. (2006). Product reviews in travel decision making. Information and Communication Technologies in Tourism 2006, 296-307.

Sun, T., Youn, S., Wu, G., \& Kuntaraporn, M. (2006). Online word-of-mouth (or mouse): An exploration of its antecedents and consequences. Journal of Computer-Mediated Communication, 11(4), 1104-1127.

The Telegraph (2014). British hotels among worst for Wi-Fi charges. Recuperado de: http://www.telegraph.co.uk/travel/destinations/europe/united-kingdom/ articles/British-hotels-among-worst-for-Wi-Fi-charges/ (21/02/2017)

TripBarometer (2015). TripBarometer March 2015, Global Travel Economy Report. TripAdvisor. Recuperado de: https://www.tripadvisor.com/TripAdvisorlnsights/ n2580/tripbarometer-2015-global-global-travel-economy (21/02/2017)

Vermeulen, I. E., \& Seegers, D. (2009). Tried and tested: The impact of online hotel reviews on consumer consideration. Tourism management, 30(1), 123-127. 